to 0.64 ; for lead (which is too soft to give good results by his method), 2.0 to 3.2. Thus in each case our values lie between his extremes. The other substances were not studied by him, and there are two among them (namely, tantalum and tungsten) which have never before had their compressibilities determined, so far as we know. It is especially satisfactory to have the apparent inconsistencies of the earlier results adequately explained and removed. ${ }^{1}$

We are greatly indebted to the Carnegie Institution of Washington for generous support in this investigation.

\title{
Summary.
}

This paper describes careful determinations involving the compressibilities of eight substances, of which two had apparently never been studied before, namely, tantalum and tungsten. The method which has been used for over ten years by one of the authors was modified by the . construction of the piezometer of steel instead of glass. This change has the advantage of avoiding the hysteresis of glass under high pressure. The results show, however, that the method of manipulation used in the earlier work had overcome this difficulty and that the earlier results were quite as satisfactory and accurate as they were supposed to be at the time. The difference in the final values of the compressibilities is due chiefly to the change in the assumed compressibility of mercury, which is now referred to a better new absolute value for iron (0.0000006I) rather than to earlier less accurate work. The compressibility of the various substances, at $20^{\circ}$ (expressed in the megabar standard, multiplied by a million) were found as follows: Copper 0.76 , lead 2.35, mercury 3.96, molybdenum 0.47 , tantalum 0.54 , tungsten 0.28 , silver bromide 2.70 . In each case the range of pressure was between 100 and 500 megabars. The outcome is as consistent as could be expected with the work of Bridgman and Grüneisen, and previous discrepancies are explained. Especial attention is called to the very small compressibility of tungsten.

Cambridge, Mass.

[CONTRIBUTION FROM THE GEOPHYSICAL LABORATORY OF THE CARNEGIE INSTITUTION.]

\section{THE MEASUREMENT OF THE FREEZING-POINT DEPRESSION OF DILUTE SOLUTIONS.}

By LEASON H. ADAMS.

Received December 20, 1914.

The present paper is a description of a method of determining the freezing-point depression of dilute solutions, by the use of which one is enabled to observe the equilibrium temperature with an uncertainty

${ }^{2}$ Attention is called to the interesting compilation by W. Schut entitled "Piëzochemie der Gecondenseerde Systemen" (Utrecht, 1912) when some of these matters are discussed. 
no greater than $0.0001^{\circ}$ and to measure the equilibrium concentration with commensurate accuracy. Results are presented for the non-electrolyte mannite and for the salts $\mathrm{KCl}$ and $\mathrm{KNO}_{3}$, all at concentrations ranging from about 5 to 100 millimolal; the latter are shown to agree very well, at the lowest concentrations, with the degree of ionization derived from conductance measurements.

The characteristic property of an electrolyte in aqueous solution is its degree of ionization, or its mol number; the latter, which is directly related to the former, is the relation of the apparent concentration-as deduced from the freezing point (or vapor pressure or osmotic pressure) of the solution by means of the laws of the perfect solution-to the concentration calculated on the basis of the respective weights of solute and solvent present. The mol-number may also be derived from the conductance ratio, on the basis of certain assumptions, of which the most important are: (I) that the equivalent conductance $\left(\Lambda_{0}\right)$ of the ions does not vary with the concentration, except in so far as the fluidity of the solution varies; (2) that an accurate value of $\Lambda_{\circ}$ can be obtained by extrapolation from the measured conductances, and that the influence of change of fluidity upon this $\Lambda_{\circ}$ value can be satisfactorily allowed for. The usual interpretation of the results obtained from any of the methods of measurement involves the assumption that the only forms in which the substance exists in the solution are the un-ionized molecules and the ultimate simple ions into which they dissociate at extreme dilution. ${ }^{1}$

Altogether then, the mol-numbers derived from the conductance ratio are trustworthy only when the uncertainties involved in the above assumptions are unimportant-in other words, only when the concentration is as small as o.I formal, or even less. Of the other methods, the one susceptible of the highest accuracy at the present time is the measurement of the freezing-point depression; but unfortunately it is a matter of some difficulty to measure the freezing point of such dilute solutions with sufficient percentage accuracy to admit of a really satisfactory comparison of the mol-numbers so derived with those derived from the conductance of such solutions. For example, a o.or formal solution of $\mathrm{KCl}$ freezes at $-0.036 \mathrm{I}^{\circ}$, so that an error of only $0.00 \mathrm{I}^{\circ}$ in the equilibrium temperature corresponds to an error of $3 \%$ on the observed lowering, and accordingly to an uncertainty of $6 \%$ in the degree of ionization derived therefrom; while further uncertainty will of course result if the concentration of the solution in equilibrium with ice at the freezing point is not known with sufficient accuracy-and lack of exactness in this respect has been a frequent source of error in the past. But for a trustworthy comparison of the mol-numbers derived in the two ways, the degree of ionization derived

${ }^{1}$ With regard to these matters, see especially A. A. Noyes and K. G. Falk, THIs JOURNAL, 32, IOII-I5 (I9I0); 34, 454-60, 487-9 (1912). 
from the freezing point should be known within I\%, even for the solutions ranging down to 0.005 formal; this degree of accuracy necessitates the determination of the freezing-point depression to $0.000 \mathrm{I}^{\circ}$, and a commensurate exactness in the equilibrium concentration. A method of achieving this accuracy is presented in the following pages; but before describing it, we shall refer briefly to some of the best recent work in this field.

During recent years the determination of the freezing-point depression of aqueous solutions has been undertaken by a large number of investigators, with a steady improvement in apparatus and technique. The principal improvements are two: the substitution of a device for measuring directly the depression of freezing point, in place of the mercury thermometer which measured the freezing point only and that with a precision probably not better than $0.001^{\circ}$; the abandonment of the old Raoult method of supercooling a solution of known concentration, which involved a correction for change of concentration due to separation of ice, as well as an uncertainty in the equilibrium temperature.

As a temperature-measuring device for accurate work, mercury thermometers were abandoned in favor of compound thermoelements by Hausrath, ${ }^{1}$ Osaka, ${ }^{2}$ Jahn, ${ }^{3}$ and Flügel; ${ }^{4}$ also by Bedford, ${ }^{5}$ who employed a differential platinum resistance thermometer. The first one to improve on the original Raoult supercooling method was Roloff, ${ }^{b}$ who, acting upon a suggestion of Nernst, determined by analysis the composition of solutions in equilibrium with a large amount of ice which had been frozen out of the solutions. Jahn, ${ }^{7}$ Fluigel, ${ }^{4}$ and Roth $^{8}$ later made use of this same method, while Walker and Robertson, ${ }^{9}$ and also Richards ${ }^{10}$ mixed the salt solutions directly with ice separately frozen and placed in the freezing-point vessel. This last procedure is to be preferred because it minimizes the chance of error from a possible solubility or inclusion of the salt in the ice.

The data of Jahn and of Flügel are probably the most generally reliable, but even they are not entirely beyond question; because, so far as one can gather, neither investigator appreciated the importance of homogeneity in wire used for thermocouples or of the necessity of special tests for, and

${ }^{1}$ H. Hausrath, Ann. Physik, [4] 9, 522 (1902).

2 Y. Osaka, Z. physik. Chem., 4I, 560 (1902).

3. Jahn, Ibid., 59, 3 I (1907).

- F. Flügel, Ibid., 79, 577 (I9I2).

s T. G. Bedford, Proc. Roy. Soc. London, (A) 83, 454 (I910).

- M. Roloff, $Z$. physik. Chem., I8, 572 (1895).

${ }^{7}$ H. Jahn, Ibid., 50, 129 (1905); 59, 3 I (1907).

8 W. A. Roth, Ibid., 79, 599 (I912).

' J. Walker and A. J. Robertson, Proc. Roy. Soc. Edinburgh, 24, 363 (1902).

${ }_{10}$ T. W. Richards, Z. physik. Chem., 44, 563 (1903); This JourNaI, 25, 29 I (I903) 
the removal of, the heterogeneous portions of the wire so used. It is also open to question whether their respective potentiometer outfits were of a type sufficiently free from parasitic electromotive forces to insure the accurate reading of small fractions of microvolts. In other words their temperature measurements may not be so accurate as the published data would suggest. This matter will be reverted to later.

The Method.-The essentials of accurate freezing-point determinations are substantially as follows: (I) Rapid and certain attainment of a (practically) stationary temperature equilibrium; (2) accurate and convenient measurement of the temperature, preferably of the freezing-point lowering directly by means of a differential method; (3) determination with sufficient exactness of the concentration of the equilibrium solution. These conditions were realized: ( $\mathrm{I}$ ) by thoroughly mixing the solution with a large amount of broken ice by means of a specially designed stirrer; (2) by measuring directly, with a multiple-junction thermoelement and sensitive potentiometer outfit, the difference between the equilibrium temperatures, ice-solution and ice-water; (3) by analyzing the equilibrium solution by means of a Zeiss interferometer ${ }^{1}$ used practically as a zero instrument. We shall now proceed to describe the apparatus in detail.

\section{Temperature Measurement.}

The Thermoelement.-Copper and constantan were selected as the thermoelectric pair since in magnitude of thermoelectric power, homogeneity, permanence, and ready availability this is, at present, the most generally suitable couple for use at low temperatures. The copper wire used was No. 36 B. S. (0.15 mm.), the constantan No. 30 (0.25 mm.) both double silk covered. ${ }^{2}$ The element was made up of 50 couples connected in series and all enclosed at each end in a single glass tube of about $7 \mathrm{~mm}$. outside diameter.

Construction of the Element.-Full details of construction are presented since they may be useful to any one desiring to make a similar element. From a sufficient length of copper, and of constantan wire which had been carefully tested for homogeneity, ${ }^{3}$ the 50 pairs of wires were cut off and covered with shellac; when dry they were bared of insulation for 2 or $3 \mathrm{~mm}$. at each end, and hard-soldered together as shown

${ }^{1}$ In the case of electrolytes the electrical conductance could well be employed as a means of determining the equilibrium concentration.

2The "Ideal" wire made by the Electical Alloy Co., Morristown, N. J., is the best constantan wire for thermoelements which we have used. Almost any copper wire will be found suitable. Instead of silk-covered wire the enameled wires now obtainable possess certain advantages, namely that the insulation is thinner and will stand more handling and a higher temperature without deteriorating.

${ }^{3}$ See W. P. White, This Journal, 36, 2296 (Igr4). The most convenient method of testing a small lot of wire is to drawn it through a narrow U-tube, preferably of metal, immersed in boiling water. It is not necessary to test the copper wire. 
diagrammatically in Fig. I, care being taken that the finished joints are free from sharp points or projections.

The best method of hard-soldering such small wires is to use a tiny gas flame ( $8 \mathrm{~mm}$. long) issuing from a glass tip with a hole $0.2 \mathrm{~mm}$. in diameter at the end; the solder consists of a mixture of 3 parts of brass to I of silver, and (anhydrous) borax glass is used as a flux. The whole operation is rapid and easy if one is careful to use small quantities of both borax and solder-not more than $0.5 \mathrm{mg}$. of borax and $3 \mathrm{mg}$. solder for each joint.

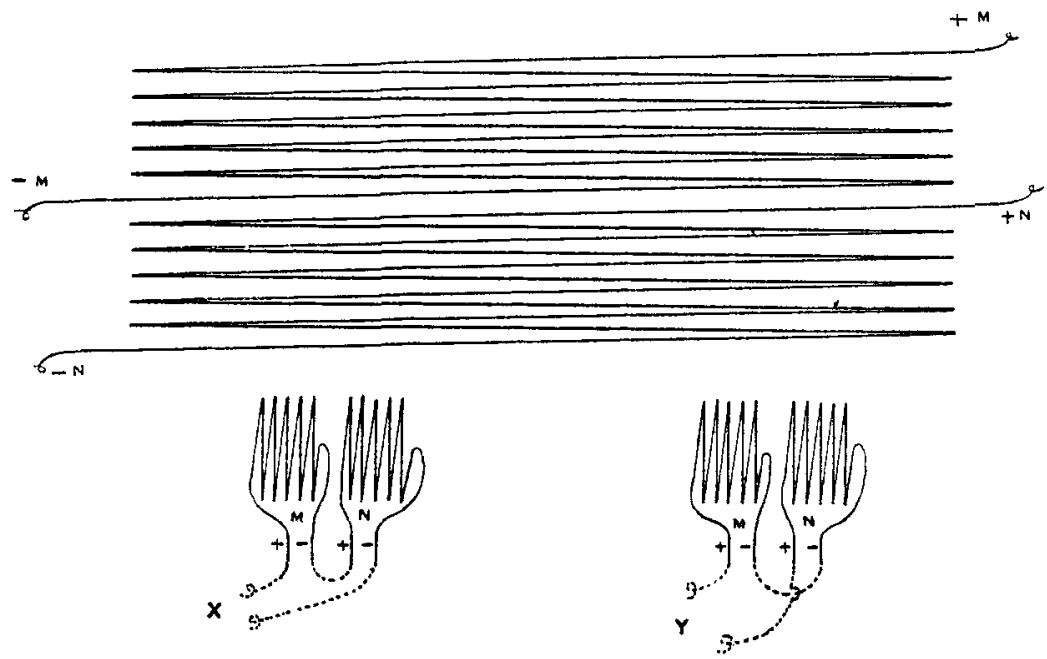

Fig. 1.-Diagram to show arrangement of the copper and constantan wires in the thermoelement. The wires are joined in two groups of 5 couples (25 in the actual element); the terminal (copper) wires of which may be connected either as at $\mathrm{X}$ or at $Y$, thus enabling one to throw the two halves either in series or in opposition.

When soldered the junctions are covered with an insulating varnish of which the best is a coating of hard rubber applied as follows: Enough precipitated sulfur, or still better insoluble sulfur, is stirred up with rather thick rubber cement (pure gum rubber dissolved in benzene and carbon disulfide) to equal $20-25 \%$ of the solid rubber. The junctions are dipped in this mixture and, after drying in the air, maintained at $140^{\circ}$ for 15 hours or longer.

The element is now ready to be assembled and enclosed. As may be seen from Fig. I the wires are joined together in two halves with four leadwires, by means of which the two halves may be connected either in series or in opposition. This arrangement allows the element to be tested at any time for electrical leaks or short circuits, since if one end is placed in ice while the other end remains at room temperature, the electromotive force at $\mathrm{Y}$ (Fig. I) will be zero only when there are no internal short circuits or leaks. Measurement of the resistance of the two halves and of 
the insulation resistance ${ }^{1}$ between them will serve the same purpose and is especially useful during the final assembling and enclosure of the wires. Experience has demonstrated the necessity of constantly testing in this way the element at all stages of construction in order to detect and obviate all internal leaks or short circuits.

In binding the wires together it is desirable to distribute the junctions in the form of a cone about $4 \mathrm{~cm}$. long and to make a compact bundle of the wires by wrapping with fine silk thread. The necessary stiffness in the central portion is secured by binding in a small brass rod, bent to shape, extending about $3 \mathrm{~cm}$. beyond each bend. Each end is embedded in fused naphthalene in a glass tube of as small diameter as practicable; and finally the central portion is paraffined (in order to exclude moisture, which may cause the insulation resistance to decrease beyond the margin of safety) and wrapped with silk ribbon. It is advisable to protect the lead-wires from possible breakage, especially at their point of exit, by twisting them together with a thin piece of cord.

Potentiometer.-A potentiometer ${ }^{2}$ of careful design was used, by means of which, in conjunction with a sensitive Weston galvanometer, a precision of 0.1 microvolt was readily attained. The whole potentiometric apparatus was protected from leakage, etc., by two equipotential shields, ${ }^{3}$ so that even in damp weather the total leakages and parasitic electromotive forces never exceeded 0.5 microvolt. The residual leakage, etc., was, however, contintously compensated for by means of the usual eliminating switch. The lowest decade consisted of steps of to microvolts each; units and tenths of microvolts were read directly with telescope and scale, the sensitiveness of the galvanometer being kept adjusted so that 1o microvolts gave a deflection of exactly ro divisions on the scale.

Calibration of the Element.-The thermoelement was calibrated against a 24 -junction element previously calibrated at the Bureau of Standards to $0.001^{\circ}$ between $0^{\circ}$ and $50^{\circ}$. Comparisons with the standard element were made in a well-stirred kerosene bath at $10^{\circ}, 20^{\circ}$, and $30^{\circ}$; from these readings an equation was calculated which expresses the relation between the temperatures as determined by the standard element and the microvolts registered by the unknown element. ${ }^{4}$ By differentia-

1 It is convenient to do this on the potentiometer itself.

2 This potentiometer has been described by W. P. White in Z. Instrumentenkunde; 34, 79 (1914).

${ }^{3}$ A full discussion of choice of potentiometers, mode of shielding, and other points in connection with potentiometer installation, is given in recent papers by $W$. P. White, This JoURNAL, 36, I856, I868, 201 I (I9I4).

- The readings of a copper-constantan couple at these low temperatures are reproduced equally well by a cubic equation of the form $E=A t+B t^{2}+C t^{3}$ and by an exponential of the form $E=A t+B\left(I-e^{C t}\right)$. At higher temperatures the latter is preferable. Compare L. H. Adams, J. Wash. Acad. Sci., 3, 469 (1913). 
tion of this equation, the gradient at $0^{\circ}$ - that is the number of microvolts per degree at $0^{\circ}$-was derived. This gradient changes by only $I / 400$ of itself per degree, and therefore small temperature differences may be accurately obtained from the readings in microvolts simply by multiplying by the reciprocal of the gradient-in the present instance $0.0005198^{\circ}$ per microvolt at $0^{\circ}$. As the temperature difference increases, however, the departure from linearity becomes appreciable, being proportional to the square of the temperature difference; thus at temperatures of $-0.3^{\circ}$, $-0.4^{\circ}$ and $-0.5^{\circ}$ the error introduced by the assumption of strict linearity is $0.0001^{\circ}, 0.0002^{\circ}$ and $0.0003^{\circ}$, respectively. The temperatures obtained by multiplying the reading in microvolts by the factor above mentioned have been corrected, therefore, by adding the appropriate small amount.

Freezing-point Apparatus. - The freezing-point vessels consist essentially of two exactly similar vacuum jacketed silvered jars $A$ and $B$ (Fig. 2), arranged so that they are completely surrounded by melting ice. One end of the thermoelement dips into $A$, which contains ice and water, the other into $B$, which contains ice and solution; thus, by means of this arrangement the thermoelement records directly the freezing-point lowering produced by the salt. Each jar is closed with a cork-stopper, previously thoroughly soaked in hot paraffin, which serves also to hold the thermoelement and pipet in position. The jars sit in cylindrical tin cans, which are supported in a large sheet metal pot, covered with a thick layer of wool felt, and nearly filled with ice. The double lid, likewise covered on the top with felt, is also filled with ice. This arrangement secures the great advantages: (I) that the maximum temperature gradient to which the thermoelement is exposed does not exceed ${ }^{\circ}{ }^{\circ} ;(2)$ that the melting of the ice, and consequently the change of concentration of the solution, and hence of freezing point, is very slow. Indeed the freezing point of the solutions, even when left unstirred, did not change by more than $0.001^{\circ}$ in an hour.

Mode of Stirring.-Thorough admixture of solution with the ice is absolutely essential; this is secured by the similar stirrers $S, S$, which are shown more in detail in Fig. 3. This stirrer is really a circulating pump, its function being to withdraw liquid from the bottom of the vessel and discharge it over the top of the ice, whence it flows downward through the ice to the bottom again; relatively few strokes are sufficient for the attainment of complete mixing and cooling. The pump is of brass, heavily gold plated. The barrel $E$ (Fig. 3 ) is a half cylinder open at both ends except for the screen at the bottom which serves to prevent ice from entering the stirrer; this barrel is held in position in the freezing vessel by means of the thin strips $H$ which act as springs. The bucket-shaped piston $D$, which is a fair fit for the inside of the barrel, is moved up and 

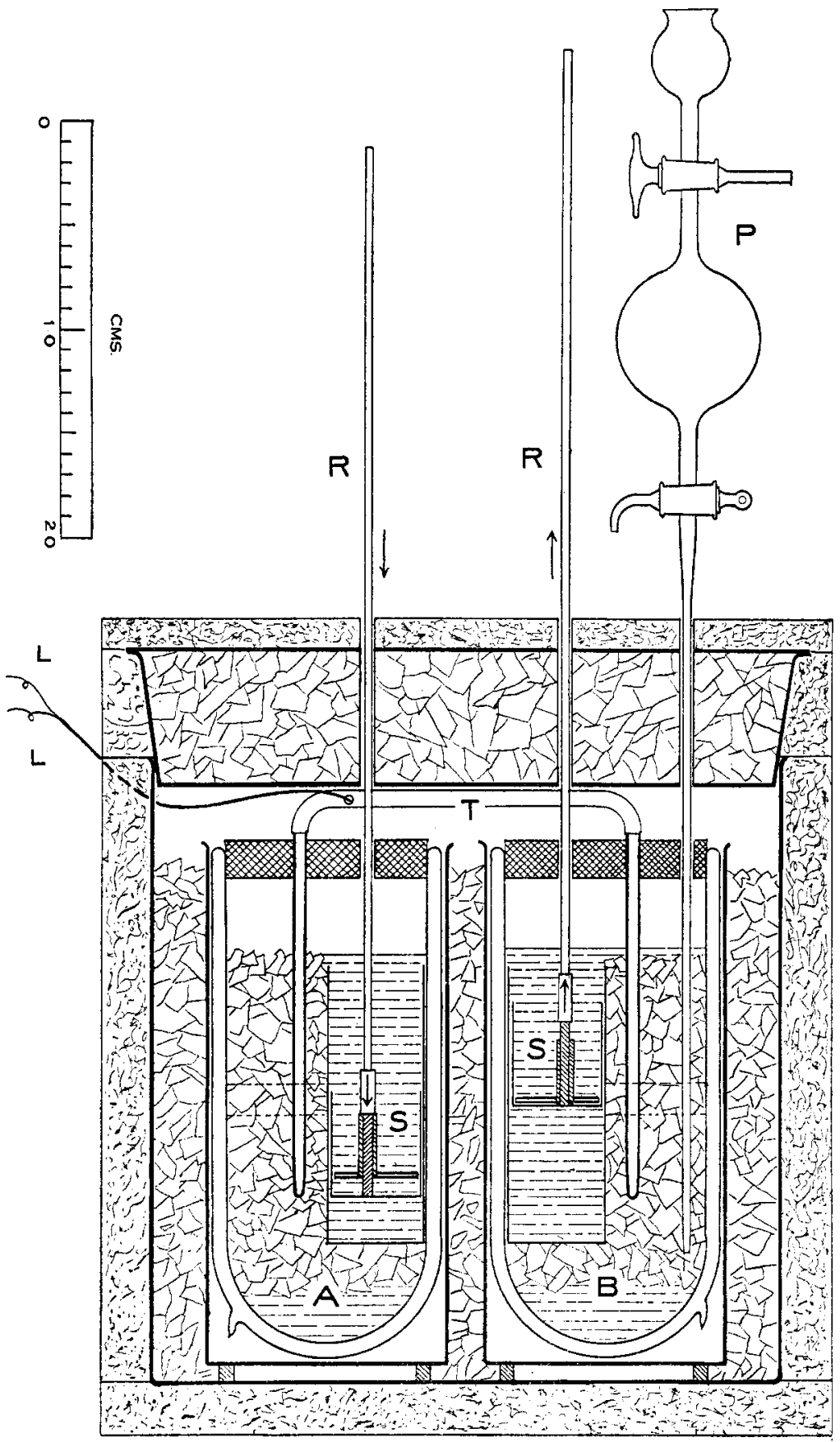
Fig, 2.-Freezing-point apparatus. Two exactly similar vacuum-jacketed vessels $(A, B)$ are surrounded with cracked ice contained in a large pot which is well insulated by a layer of wool felt. The double lid is also filled with ice and covered with felt. Jar A contains finally about $400 \mathrm{~g}$. ice and $600 \mathrm{~g}$. water; jar B the same respective amounts of ice and solution. The liquid in each jar is stirred by means of the similar hemicylindrical stirrers $S, S$ which, when actuated by the glass rods $R, R$, pump liquid from the bottom of each jar and discharge it at the top over the ice. The difference in temperature between-ice-water and ice-solution is measured directly by the differential thermoelement of 50 junctions and sensitive to $0.0001^{\circ}$. Two thermoelement lead-wires (there are really four) are shown at $L, L$. When temperature equilibrium is attained a sample for analysis is withdrawn by means of the combined pipet and funnel $P$, which also serves to introduce water and solute into jar $B$. When equilibrium is attained in both $\mathrm{A}$ and $\mathrm{B}$, the temperature difference to $0.000 \mathrm{I}^{\circ}$ is measured directly by means of the 50 -junction differential thermoelement ( $T$; its terminal wires are $L, I)$; the concentration is determined on a sample withdrawn through the pipet $P$.

down by means of a glass rod fastened to the central stem, $G$; it is open at the top, and its bottom is slotted as shown in the figure. These open
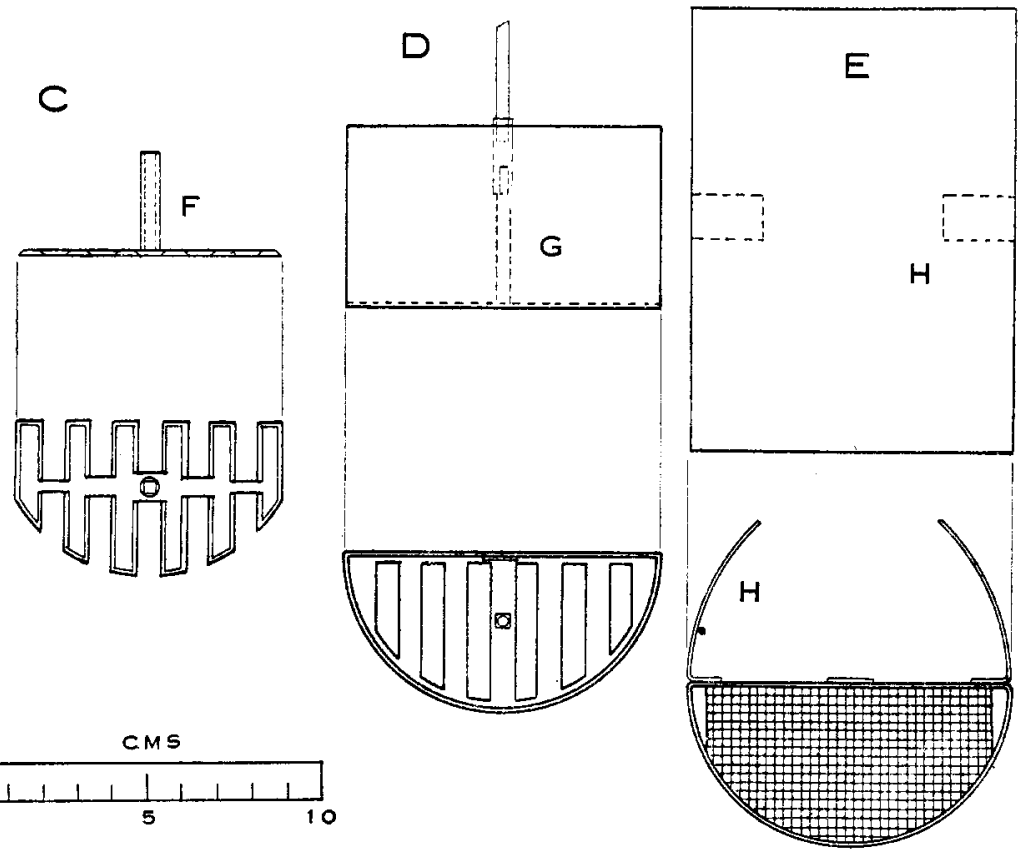

Fig. 3.-Drawing in plan and elevation of stirrer; to show mode of operation. The stirrer, made of brass heavily gold-plated, is really a circulatory pump with a hemicylindrical barrel. In this barrel, $\mathrm{E}$, open at both ends, the bucket-shaped piston $D$ slides easily; $D$ is open at the top and slotted at the bottom, the slots being closed by the clapper $C$ when $D$ is raised, but open when $D$ is lowered. The clapper $C$ is kept in alignment by the bearing $F$ which slides on the square stem $G$. The stirrer is operated by means of the glass rod R (Fig. 2), and held in place in the freezing-point vessel by the springs $H, H$. 
spaces are just covered by the solid portions of the clapper-valve $C$ which is held in alignment by the small hollow barrel $F$ sliding on the stem $G$. It is thus evident that when the bucket with clapper in place is pushed down through liquid contained in the barrel, the pressure of the liquid from below will force the clapper to rise and allow liquid to flow through; but when the bucket is raised the clapper is held down, and hence liquid is lifted up and out over the top of the barrel. The piston was made bucket-shaped in order to avoid any considerable leak and to have at the same time a loose and easy fit. These stirrers proved to be very efficient; each stroke circulated approximately one-half of the liquid in the vessel, and consequently but little stirring was required.

Analysis of the Equilibrium Solution.-Samples for analysis were withdrawn by means of the sampling pipet $P$, which served also for the addition of water or of solute (in the form of a concentrated cooled solution). The concentration of the solutions was determined by what is practically a zero method; namely, a Zeiss interferometer was used to compare this unknown solution with known solutions of very nearly the same concentration, and hence served practically as a means of interpolation over a small range. In the case of mannite a series of known solutions was compared with water, and the readings plotted; the unknown solutions were also compared with water and their concentration was derived by interpolation on this curve of the readings of the instrument. The former way is in general preferable, since there is then less chance of mistaking the proper band for comparison.

With the instrument used in this work ${ }^{1}$ one can bring the upper and lower bands into conjunction with an uncertainty of not more than $I$ division on the scale. It is thus possible, with the $40 \mathrm{~mm}$. chamber, to determine the percentage composition of a solution of a colorless salt or non-electrolyte to within 0.0002 - that is to within two parts of salt in a million of water. The attainment of this degree of certainty demands some experience with the instrument; this matter will not be discussed further here, since it forms the subject of another communication.

Experimental Procedure.-The vacuum-jacketed jars are set in position in the large can and surrounded by cracked ice; the stirrers are set in place and about $500 \mathrm{cc}$. of cooled distilled water poured into each jar. Ice, ${ }^{2}$ in pieces about the size of an almond and previously washed with distilled water, is then put into each vessel until the half not occupied by the stirrer is filled; this required about $500 \mathrm{~g}$. The water level is then adjusted so as to be about $5 \mathrm{~mm}$. above the top of the stirrer. The paraf-

1 The Water Interferometer manufactured by Carl Zeiss, Jena.

2 Ordinary artificial ice was used. This ice was always very pure, being made from distilled and filtered water. Moreover, as will be explained below, any small residual impurity in the ice would be without prejudical effect on the final results. 
fined cork stoppers are then placed on the jars, one leg of the thermoelement pushed into each $\mathrm{jar}$ and the ice-filled cover placed in position ${ }^{1}$ on the outer can. The narrow tube of the sampling pipet is then shoved through a hole in the cover and down into jar $B$.

The apparatus is then allowed to stand for two or three hours with occasional stirring in order that all the various parts in the interior may cool down to nearly zero; after which both sides are stirred until a constant thermoelement reading is obtained. This so-called "zero" reading ${ }^{2}$ is noted, and a sample of about $50 \mathrm{cc}$. withdrawn from jar $B$ and preserved in a Jena glass flask sealed with a sheet of tinfoil tightly crimped about the neck of the flask. The purpose of this initial sample is to determine a "concentration zero" reading on the interferometer. The desired amount of solute dissolved in enough ice-cold water to make up the exact volume of liquid previously withdrawn is then added; both sides are again stirred at intervals until temperature equilibrium is attained. In order to rinse the pipet $P$, it is half filled with solution by suction and given a gentle rotary shake, after which process the solution is returned to the vessel. A few strokes of the stirrer suffices to bring about a condition of equilibrium which, with occasional stirring, does not change in the course of several minutes. The temperature is now read and a sample of $5^{\circ} \mathrm{cc}$. for analysis withdrawn by means of the pipet $P$. More salt may now be introduced for a new determination. From each temperature reading the "zero" temperature reading is subtracted and similarly from each concentration reading is deducted the "zero" concentration reading - that is, the reading on the interferometer of the zero sample against the water from which the standard calibration solutions were made up.

Usually a series of five or six determinations was carried out with one set-up of the apparatus; but it is to be noted that the several pairs of readings constitute a series of independent observations. That there is no serious systematic error follows from the smallness and constancy of the two zero readings; moreover, any factor prejudicial to the result would have increased both of these readings and hence would be substantially eliminated. Nevertheless, it was thought desirable to have a direct check on the accuracy of the method; for this purpose a series of determinations were made with dilute solutions of the non-electrolyte mannite. These results, together with those obtained with the salts potassium nitrate and potassium chloride, will now be presented.

1 This was facilitated by guiding rods (not shown in Fig. 2).

2 This reading was usually about I microvolt, but probably does not indicate a corresponding difference in temperature $\left(0.0005^{\circ}\right)$ between the two vessels. It undoubtedly represents the summation of several small electromotive forces. The analogous concentration "zero" reading was never greater than 3 divisions on the interferometer. 


\section{Experimental Results.}

Mannite.-The mannite was recrystallized from water and dried by heating in vacuo for several hours at $130^{\circ}$. Table I is a complete record of the observations. The first three columns reproduce (I) the measured equilibrium concentration $\left(N \times 10^{3}\right)$, expressed in millimols per Iooo $\mathrm{g}$. of water, and the directly measured freezing-point depression (II) in microvolts and (III) in degrees. The fourth column shows the calculated lowering as derived from the equation $N=\Delta t(\mathrm{I}+0.0055 \Delta t) / \mathrm{r} .858$, the theoretical expression for the depression of a solution containing $N$ moles per rooo g. of water; the fifth gives the differences, in ten-thousandths of a degree, between the observations and the values so calculated. The last two columns are a similar comparison of the observed concentration with that calculated from the observed depression.

The differences are in no case greater than $0.0001^{\circ}$, and thus justify our expectations as to the accuracy of the method.

Table I.-Freezing-Point Depressions Produced by Mannite in Difute Aqueous

I.
Concentration
millimols per
1000 g. $\mathrm{H}_{2} \mathrm{O}$.
$\mathrm{N} \times 10^{3}$
4.02
8.42
14.04
28.29
62.59

\begin{tabular}{cc}
$\begin{array}{cc}\text { Ir. } \\
\text { F.-p. depression. } \\
\Delta t \text { (obs.). }\end{array}$ \\
\hline$\mu v$. & Degrees. \\
14.4 & 0.0075 \\
30.3 & 0.0157 \\
50.0 & 0.0260 \\
100.9 & 0.0525 \\
223.5 & 0.1162
\end{tabular}

SOLUTION.

Potassium Nitrate.-This salt was chosen for the reason that, as shown in the compilation of Noyes and Falk, ${ }^{1}$ there is a large discrepancy between the ionization values of $\mathrm{KNO}_{3}$, as determined by the two methods of freezing-point lowering and conductance ratio.

The salt used was twice recrystallized from $\mathrm{H}_{2} \mathrm{O}$ and dried by heating in vacuo at $150^{\circ}$ for several hours. The results are given in Table II. Table II.-FreEzing-Point Depressions Produced by $\mathrm{KNO}_{3}$ in Dilute Aqueous

\begin{tabular}{|c|}
\hline $\begin{array}{c}\text { I. } \\
\text { Concentration } \\
\text { formula-weights } \\
\text { per } 1000 \mathrm{~g} . \mathrm{H}_{2} \mathrm{O} \\
\mathrm{N} .\end{array}$ \\
\hline 0.00443 \\
\hline 0.00817 \\
\hline 0.01565 \\
\hline 0.03874 \\
\hline 0.05766 \\
\hline 0.1227 \\
\hline
\end{tabular}

\begin{tabular}{rrrr}
\multicolumn{1}{c}{ II. } & III. & \\
\hline$\mu \nu$. & & Degrees. & IV. \\
30.9 & & $0.016 \mathrm{I}$ & 3.63 \\
56.8 & 0.0295 & 3.61 \\
107.4 & & $0.055^{8}$ & 3.565 \\
258.3 & & 0.1343 & 3.467 \\
$377 . \mathrm{I}$ & 0.1960 & 3.399 \\
772.4 & & 0.4017 & 3.274
\end{tabular}

The first column exhibits the measured concentration $(N)$, in formulaweights per $1000 \mathrm{~g}$. water, the second and third the observed depression

${ }^{1}$ Noyes and Falk, This JourNaL, 34, 485-9 (IgI2). 
in microvolts and degrees, respectively; the last column contains the several values of $\Delta t / N$, the formal lowering. The concordance between the several observations is shown in Fig. 4 , in which the formal lowering is plotted against $\log 10^{3} N ;$ the dotted lines above and below the curve indicate the distance through which the curve would be displaced in either

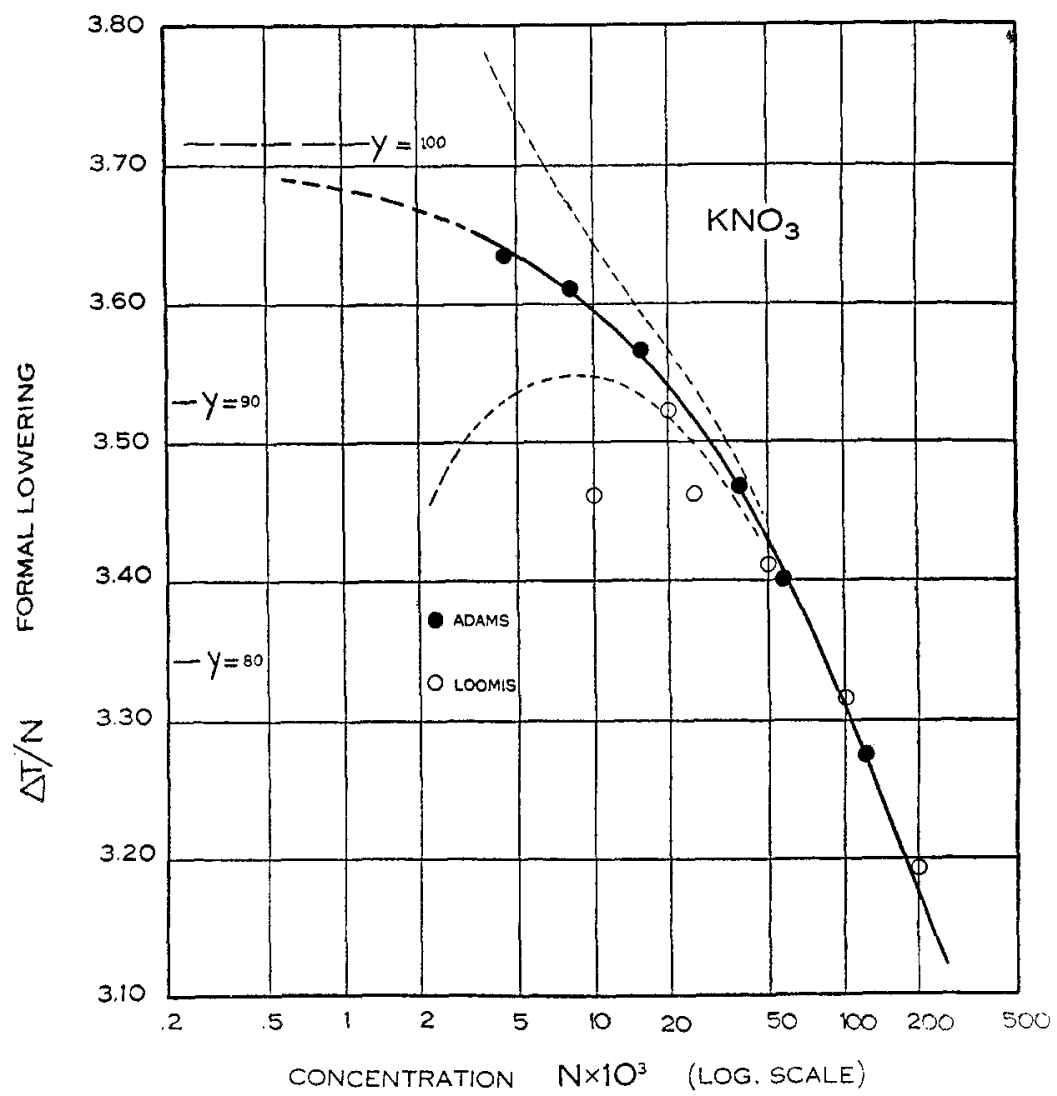

Fig. 4.-Observations on the freezing-point depression produced by $\mathrm{KNO}_{8}$ in dilute solution. The dotted lines above and below the curve represent the displacement of the curve which would be caused by an error of $\pm 0.0005^{\circ}$.

direction by an error of $0.0005^{\circ}$. For the sake of comparison the data of Loomis ${ }^{2}$ are also included; his results are in general too low, as Noyes and Falk ${ }^{3}$ presumed. The recent measurements of Roth, ${ }^{4}$ made by a method identical with that used by Loomis, are so numerous that they

1 This diagram was actually plotted on coördinate paper having a logarithmic scale in one direction.

E. H. Loomis, Ann. Physik, 57, 504 (1896).

'Noyes and Falk, This Journal, 32, IOI7 (I9Io).

+W. A. Roth, Z. physik. Chem., 79, 599 (Igr2). 
could not well be included in the diagram; Roth's data lie very close to those of Loomis and are, therefore, presumably affected by a similar error.

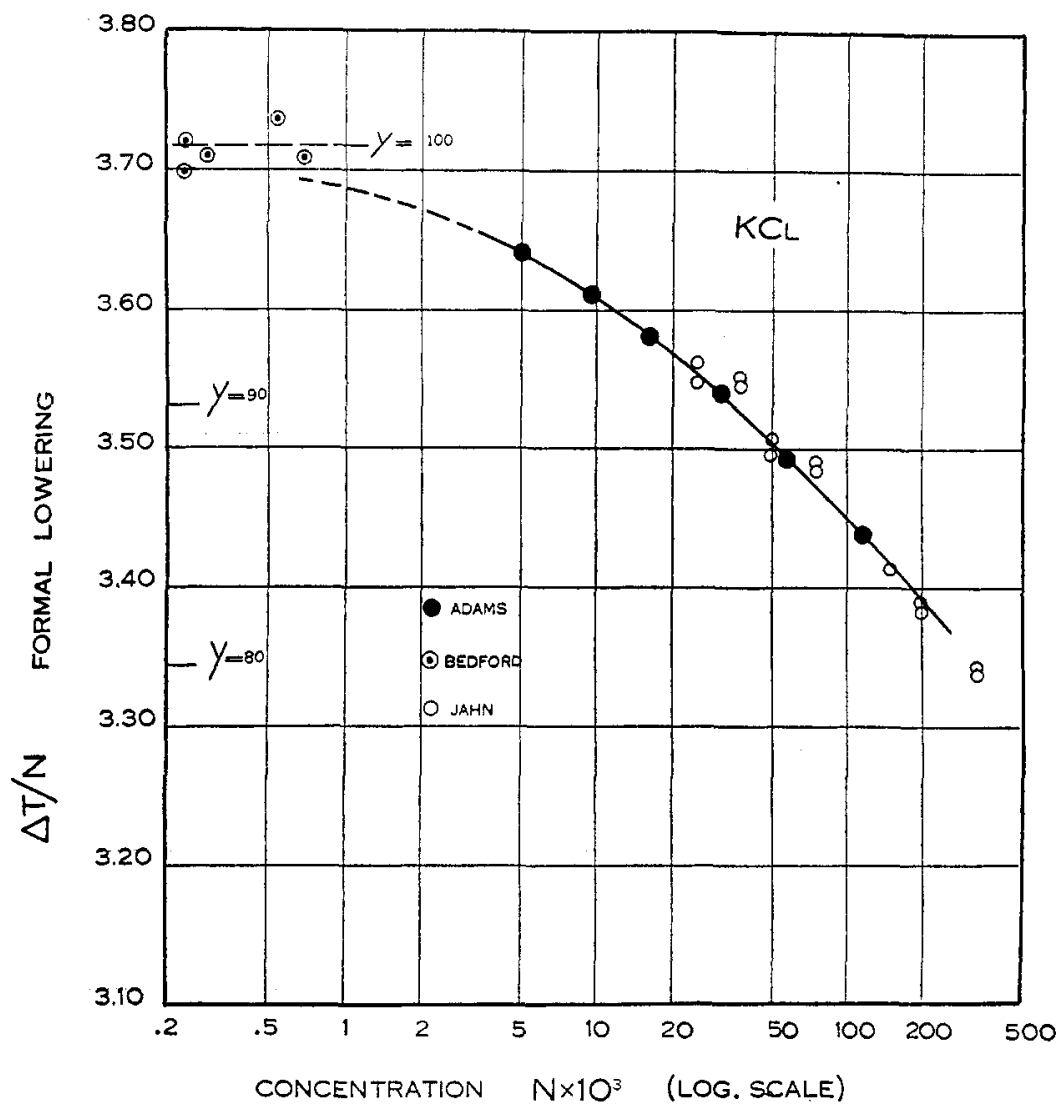

Fig. 5.-Observations on the freezing-point depression produced by $\mathrm{KCl}$ in dilute solution.

Potassium Chloride.-This salt was twice recrystallized from $\mathrm{H}_{2} \mathrm{O}$, dried first in vacuo and then ignited in a platinum dish at a temperature Table III.-Freezing-Point Depressions Produced ay KCl in Dilute Aquious

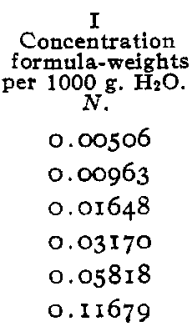

Solution.

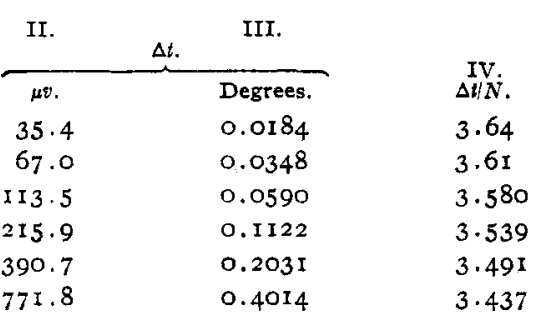


just below its melting point. The observations are brought together in Table III and plotted in Fig. 5, in which are also included the data of $\mathrm{Jahn}^{1}$ and Bedford ${ }^{2}$ on this salt.

Discussion of Results.-From the observations tabulated in Tables II and III values of the formal lowering at round concentrations were obtained by graphic interpolation; these are presented in Table IV. The first column contains the concentration $(N)$; the second, the corresponding formal lowering; the third, the mol-number $(i)$ as calculated from the equation $i=\Delta t(1+0.0055 \Delta t) / 1.858 N$, and the fourth, the corresponding percentage ionization $\gamma$ as derived from the relation $\gamma=$ $100(i-\mathrm{I})$.

The ionization values in Cols. V and VI are taken from the paper of Noyes and Falk; ${ }^{3}$ they are derived, respectively, from the conductanceviscosity ratio and from the weighted means of the freezing-point data on these salts available in I9Io.

Table IV.-Formal Lowerings at Round Concentrations, and the Derived Mol.-Numbers and Ionization Values; Comparison with the ConDUCTANCE RATIO.

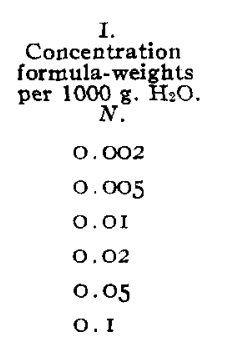

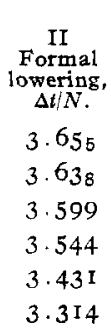

$\mathrm{KNO}_{3}$.

$\begin{array}{llllll}0.002 & 3.658 & \mathrm{I} .96_{6} & 96.9 & 97 . \mathrm{I} & \ldots \\ 0.005 & 3.643 & 1.96_{1} & 96.1 & 95.6 & 96.3 \\ 0.01 & 3.609 & \mathrm{I} .943 & 94.3 & 94 . \mathrm{I} & 94.3 \\ 0.02 & 3.570 & 1.922 & 92.2 & 92.2 & 91.8 \\ 0.05 & 3.505 & 1.888 & 88.8 & 88.9 & 88.5 \\ 0.1 & 3.45 \mathrm{I} & \mathrm{I} .861 & 86.1 & 86.0 & 86 . \mathrm{I}\end{array}$

Comparison of Cols. IV and V shows that in the case of potassium nitrate the difference between the ionization derived from freezing point and from conductance is no greater than the experimental error at all concentrations up to 0.02 molal; this disposes of the discrepancy noted by Noyes and Falk, whose mean values for this salt are derived solely from Loomis' data which, as they considered probable, are too low. At the higher concentrations the values in Cols. IV and V diverge; this shows merely

I H. Jahn, Z. physik. Chem., 50, I37 (1905).

2 T. G. Bedford, Proc. Roy. Soc. London, $(A) 83,454$ (1910).

${ }^{3}$ Noyes and Falk, This Journal, 34, 486 (IgI2). 
that some of the assumptions implicit in these calculations are not fulfilled. ${ }^{1}$ In the case of potassium chloride the agreement between the several values of the percentage ionization is all that could be desired $;^{2}$ this serves to demonstrate the trustworthiness of the method here described, since the mean difference between these new observations and the weighted mean-in this case of a large number of (somewhat discordant) results published by eleven different authors-is no greater than $0.0001^{\circ}$ (or the corresponding amount in terms of concentration).

It is thus evident that by the proper choice of method and apparatus it is possible to determine freezing-point depressions with an accuracy of $0.0001^{\circ}$. Indeed there appears to be no inherent reason why it should not be practicable to attain a tenfold accuracy by the use of a still more sensitive differential temperature measuring device, and of a still more refined means of determining the concentration of the equilibrium solution, both of which appear from our experience to be within the limits of practicability.

\section{Summary.}

Trustworthy values of the mol-number and of the corresponding ionization value can be derived from observations of the depression of freezing point only when the concentration, as well as the temperature, of the solution in equilibrium with ice have been determined with sufficient, and commensurate, accuracy. In the work described in the preceding pages the depression was measured directly with an uncertainty of not more than $0.0001^{\circ}$ by means of a 50 -junction copper-constantan differential thermoelement (which gives 2000 microvolts per degree) and a potentiometric system which enabled the electromotive force to be read to o. I microvolt. A prerequisite for the attainment of this degree of accuracy is a condition of stationary equilibrium, which was secured by thorough mixing of the solution with a large quantity of ice in a vacuum-jacketed vessel completely surrounded by melting ice. The concentration of the equilibrium solution was determined by a zero method in which a Zeiss interferometer was used as a means of comparison; the sensitiveness of this method is about 2 parts of solute per million of water.

Results are presented for solutions of the non-electrolyte mannite and of the salts potassium nitrate and chloride at concentrations ranging from 0.004 to 0.1 formula weights per $1000 \mathrm{~g}$. water; consideration of these shows that the method is susceptible of the accuracy claimed for it.

WAshingtoN, D. C.

${ }_{1}$ Cf. Noyes and Falk, Ibid., 34, 488 (I912).

${ }^{2}$ Kraus and Bray (This Journal, 35, I4I2 (IgI3)) conclude that the value of $\Lambda_{0}$ for $\mathrm{KCl}$ is 128.3 at $18^{\circ}$; on this basis the ionization values given in Col. $\mathrm{V}$ of Table IV should be increased in the ratio $130 / 128.3$ and the agreement with Col. IV would then be less satisfactory. 